\title{
Aspetos relacionados à qualidade de vida e atividade física de policiais militares de Santa Catarina - Brasil
}

\author{
Aspects related to quality of life and physical activity of military police \\ officers of Santa Catarina - Brazil
}

\author{
R. Silva, A.M. Schlichting, J.P. Schlichting, P.J. Gutierres Filho, F. Adami, A. Silva
}

ARTIGO ORIGINAL | ORIGINAL ARTICLE

\begin{abstract}
Este estudo teve como objetivo investigar a relação entre qualidade de vida, saúde, atividade física, ocupação, composição corporal e características sócio-demográficas de Policiais Militares do Estado de Santa Catarina, Brasil. Participaram 302 Policiais Militares da região metropolitana da Capital do Estado de Santa Catarina, Brasil, selecionados aleatoriamente através de sorteio simples e que atenderam aos critérios de elegibilidade. Os dados foram coletados através do Questionário da Organização Mundial de Saúde sobre Qualidade de Vida - versão curta (WHOQOL-Bref) e do Questionário da Organização Mundial de Saúde sobre Atividade Física - versão longa (IPAQ-Long), além de uma planilha sobre variáveis sócio-demográficas, antropométricas, ocupacionais e de saúde. Constatou-se que a maioria dos Policiais Militares é casada, escolarizada e com média de 36.6 anos de idade e 15.1 anos de atuação policial, apresenta boa percepção de qualidade de vida, possui níveis de atividade física acima do recomendado, está na faixa de peso recomendado e tem boa saúde. Pode-se concluir que existem associações da qualidade de vida com as atividades físicas de lazer, a estatura e a situação marital.

Palavras-chave: qualidade de vida, saúde, atividade motora, composição corporal, policial
\end{abstract}

ABSTRACT

This study aims to investigate the relationship between quality of life, health, physical activity, occupation, body composition and sociodemographic characteristics of Military Police of Santa Catarina, Brazil. 302 Police Officers participated in this study, randomly selected through a simple drawing and in accordance with the eligibility criteria. Data were collected through two questionnaires from the World Health Organization, one of them concerning quality of life (WHOQOL-Bref), and the other, concerning physical activity (IPAQ-Long), and additionally, a worksheet containing sociodemographic, anthropometric, occupational and health-related variables. It was found that most of the Military Police is married, schooled, with an average of 36.6 years old and 15.1 years of the police action, has good perception for quality of life, has levels of physical activity above the recommended, has weight in the range recommended, and has good health. It can be concluded that there are associations of quality of life with leisure physical activities, height and marital status.

Keywords: quality of life, health, motor activity, body composition, police

Submetido: 17.07.2011 | Aceite: 24.08.2012

Rudney da Silva e Paulo José Barbosa Gutierres Filho. Laboratório de Atividade Motora Adaptada, Centro de Ciências da Saúde e do Esporte, Universidade do Estado de Santa Catarina, Florianópolis, Brasil.

Antônio Melo Schlichting e Jader Peron Schlichting. Secretaria de Segurança Pública, Governo do Estado de Santa Catarina, Florianópolis, Brasil.

Fernando Adami. Faculdade de Saúde Pública, Programa de Pós-graduação em Saúde Pública, Universidade de São Paulo, São Paulo, Brasil.

António Silva. Universidade de Trás-os-Montes e Alto Douro. Centro de Investigação em Desporto, Saúde e Desenvolvimento Humano, Vila Real, Portugal.

Endereço para correspondência: Rudney da Silva, Laboratório de Atividade Motora Adaptada, Centro de Ciências da Saúde e do Esporte, Universidade do Estado de Santa Catarina, Rua Pascoal Simone, 358, Coqueiros, CEP 88080-350, Florianópolis, SC, Brasil.

E-mail: rudney.silva@udesc.br 
A expressão qualidade de vida surgiu em 1920 com as discussões sobre Estado, economia e bem-estar material de indivíduos de classes sociais menos favorecidas (Sirgy, 2001). O termo qualidade de vida passou a ter maior difusão após a Segunda Guerra Mundial e visava o entendimento do modo de vida dos indivíduos e seu suporte financeiro. Nas últimas décadas, o conceito de qualidade de vida tem sido associado principalmente à saúde humana, constituindo-se assim, relevante aspecto para estudo e melhoria da saúde pública atual (Meeberg, 1993).

A qualidade de vida pode ser definida como a percepção particular da posição do indivíduo na vida e no contexto da cultura, bem como nos sistemas de valor que são vivenciados e associados às suas metas, expectativas, padrões e preocupações, sendo, portanto, um fenômeno de percepção pessoal que denota o modo como o indivíduo sente a sua saúde e outros estados associados, como bem-estar e ocupação (Farquhar, 1995; Gill, 1994).

O trabalho humano destaca-se como um dos fatores significativos à qualidade de vida, principalmente por suas consequências à saúde dos indivíduos. Entretanto, estas consequências são diferentes entre os níveis de desenvolvimento econômico e social específicos a cada região, pois nos países com pouco desenvolvimento, prevalecem os problemas de saúde associados à fome, ao saneamento básico e às doenças transmissíveis, enquanto nos países com acentuado desenvolvimento, predominam as doenças não transmissíveis (Forattini, 1992; Leavell \& Clark, 1978).

As doenças não transmissíveis estão associadas ao estilo de vida das sociedades industrializadas. As influências deletérias do trabalho na atualidade referem-se principalmente à diminuição do tempo livre ao lazer e à família, à deterioração do poder econômico, à restrição de acesso a bens e serviços essenciais, à exposição a agentes stressores, e ao sedentarismo (Sallis \& Owen, 1999).
Algumas profissões acentuam estas condições ocupacionais deletérias, como, por exemplo, a de militares (Smith et al., 2007). No Brasil, considera-se a polícia como uma força auxiliar e reserva às forças armadas, que tem como missão a polícia ostensiva e a preservação da ordem pública. Esta instituição baseia-se na disciplina e na hierarquia militar e aplica os limites de sua competência na ação ostensiva, preventiva e repressiva com o objetivo da segurança integrada de defesa territorial e civil (Polícia Militar de Santa Catarina, 2008).

Estas ações inerentes à atuação policial impõem exigências fisiológicas, recorrências de estados distressores, rotinas extenuantes e cargas psíquicas que provocam danos físicos e psíquicos a estes profissionais (Berg, Hem, Lau, \& Ekeberg, 2005; Deschamps, Paganon-Badinier, Marchand, \& Merle, 2003). Entre os danos psíquicos, pode-se apontar a dependência alcoólica, os abusos no uso de medicamentos, a depressão e os transtornos de diversos tipos, tais como a desordem de stress pós-traumático ou síndrome de exaustão emocional ou burn-out, entre outros (Loo, 2004). Já entre os danos físicos, podem ser citadas as doenças cardiovasculares e as doenças metabólicas, entre outras (Sorensen, Smolander, Louhevaara, Korhonen, \& Oja, 2000).

Deste modo, este estudo teve como objetivo investigar a relação entre qualidade de vida, saúde, atividade física, ocupação, composição corporal e características sócio-demográficas de Policiais Militares do Estado de Santa Catarina, Brasil.

\section{MÉTODO}

Este estudo foi caracterizado como uma pesquisa do tipo analítico transversal (Pereira, 2002; Thomas \& Nelson, 2001). Todos os procedimentos foram previamente aprovados pelo Comitê de Ética em Pesquisas Envolvendo Seres Humanos da Universidade do Estado de Santa Catarina (Parecer 177/2007), respeitando à legislação brasileira e atendendo às recomen- 
dações éticas internacionais (Resolução 196, 1996; Diniz \& Corrêa, 2001; World Medical Association, 1997).

\section{Amostra}

A corporação da polícia militar do Estado de Santa Catarina é composta de 16.502 sujeitos, sendo que 11.663 encontram-se na ativa $(\mathrm{N})$. O cálculo amostral determinou a necessidade de 397 sujeitos de pesquisa (n) obtidos aleatoriamente por sorteio simples, com erro amostral tolerável de $5 \%$, nível de confiança de $95 \%$ de precisão (Barbetta, 2003).

A amostra foi composta por Policiais Militares das cinco grandes regiões do Estado de Santa Catarina, Brasil, que atenderam aos critérios de inclusão e de exclusão. Deste modo, foram incluídos os Policiais Militares que apresentassem intenção voluntária integral para participar da pesquisa, que fossem do gênero masculino, possuíssem idade superior a 18 anos e estivessem na categoria ativa da corporação. Foram excluídos da amostra os Policiais Militares afastados por motivo de doença.

A composição final da amostra apontou 302 indivíduos, com idade mínima de 19 anos, máxima de 51 anos, média de 36.6 anos e mediana de 42 anos. Este quantitativo foi obtido após recolhidos os questionários e procedidas a tabulação e análise detalhadas dos resultados, verificando-se que 13 questionários não foram devolvidos, 13 questionários eram de sujeitos do gênero feminino e 69 questionários não tinham a integralidade dos dados preenchidos, validando-se 302 questionários e estabelecendo uma taxa de resposta de aproximadamente $76 \%$.

\section{Instrumentos e Procedimentos}

Foram utilizados neste estudo dois instrumentos da Organização Mundial de Saúde: (1) Questionário sobre Qualidade de Vida, versão curta (WHOQOL-Bref) (Fleck, Leal, Louzada, \& Xavier, 1999; Fleck, Louzada, \& Xavier, 2000; Harper \& Power, 1998); (2) Questionário sobre Atividades Físicas, versão longa (IPAQ-Long) (Craig et al., 2003; Matsudo, Araujo, \& Matsudo, 2001; Pardini, Matsudo, \& Araújo, 2001).

Além destes instrumentos, foi utilizada ainda uma planilha para coleta de dados sobre fatores sócio-demográficos, ocupacionais e antropométricos. Os fatores sócio-demográficos incluíram gênero, idade, grau de instrução e estado civil. Os fatores ocupacionais incluíram tempo de serviço, tipo de atuação e realização de trabalhos extra. Os fatores antropométricos incluíram peso e estatura corporal.

O WHOQOL-Bref é composto de 26 questões que investigam a qualidade de vida em quatro domínios: físico, psicológico, social, ambiental (Fleck et al., 1999; Fleck et al., 2000; Harper \& Power, 1998). O IPAQ-Long é composto de 25 questões que avaliam a atividade física a partir de cinco domínios: trabalho, tarefas domésticas, transporte, lazer/ esporte/recreação, e de tempo sentado (Craig et al., 2003; Matsudo et al., 2001; Pardini et al., 2001).

A coleta dos dados foi iniciada somente depois da recolha do consentimento livre e informado dos sujeitos de pesquisa, adotando-se cuidados adicionais para minimizar os riscos envolvidos na pesquisa com populações vulneráveis, procedendo-se à identificação nos instrumentos somente por códigos estabelecidos aleatoriamente e de acesso exclusivo dos pesquisadores (Brazil, 1996).

Os dados foram coletados diretamente no local de trabalho dos Policiais Militares através da entrega e recolha posterior dos instrumentos sem identificações aparentes. Foram disponibilizados contatos telefônicos e de correio eletrônico, além de visitas aos batalhões, para esclarecimentos relacionados à pesquisa, já que os pesquisadores tinham permissão para acesso aos locais de pesquisa. O tempo médio entre a entrega e a recolha foi de aproximadamente 72 horas.

Todas as análises estatísticas foram reali- 
zadas no programa SPSS, versão 17.0. A confiabilidade dos resultados do WHOQOL-Bref e do IPAQ-Long foi realizada através do cálculo do coeficiente Cronbach, adotando um valor de alfa igual ou superior a 0.70 tanto para a totalidade dos itens avaliados, quanto para apenas os itens estandardizados.

Os dados das variáveis antropométricas, demográficas, ocupacionais, de atividade física e de qualidade de vida foram primeiramente analisados por meio de estatística descritiva. A relação entre os valores de qualidade de vida e variáveis preditoras foi analisada primeiramente sob modelo de regressão linear simples e posteriormente sob modelo múltiplo. Dessa forma, a variável dependente considerada foi o escore total obtido do WHOQQL-Bref, com as variáveis demográficas, antropométricas, ocupacionais e de atividade físicas consideradas como independentes. Cada variável independente foi analisada em relação à dependente segundo um modelo de regressão linear simples, sendo o ponto de corte para entrada no modelo múltiplo o valor de $p$ menor ou igual a 0.20. Para modelo múltiplo, as variáveis foram sendo imputadas segundo modelo forward, sendo consideradas significantes quando o $p$ fosse menor que 0.05 e/ou quando modificassem em mais de $10 \%$ o valor do coeficiente angular $(\beta)$ de qualquer variável do modelo. $\mathrm{O}$ modelo múltiplo pode ser descrito segundo a equação:

$$
Y=\beta_{0}+\beta_{1} X+\beta_{2} X+\ldots+\beta_{\mathrm{n}} X
$$

\section{Onde:}

$Y=$ valor predito da variável dependente;

$\beta_{0}=$ intercepto do eixo $Y$ (valor da variável $Y$, quando $X=0$ );

$\beta_{\mathrm{i}}=$ inclinação da reta (coeficiente angular - valor do incremento de $Y$ para cada unidade de $X$, quando todas as outras variáveis independentes permaneçam constantes);

Os valores de referência do I-PAQ-Long foram calculados segundo diretrizes do Instituto Karolinska da Suécia (Karolinska Institutet,
2005) e os valores de referência do WHOQOL-Bref calculados conforme critérios da Organização Mundial de Saúde (World Health Organization, 1996), através de sintaxes construídas para classificação do nível de atividade física em baixo, moderado e alto, e da qualidade de vida e seus domínios em escores variáveis entre 0 (menor índice) e 100 pontos (maior índice). Os valores de classificação do índice de massa corporal foram calculados com base nos critérios da Organização Mundial de Saúde (World Health Organization, 1995). Os valores da classificação dos sujeitos quanto aos indicadores de risco e seus impactos à saúde foram calculados de acordo com a escala proposta pelo Instituto para Pesquisa Social do Canadá (Institute for Social Research, 2005). Os valores referentes à estatura corporal foram agrupados a cada $10 \mathrm{~cm}$ e os valores do peso corporal foram agrupados a cada $10 \mathrm{~kg}$.

\section{RESULTADOS}

\section{Análise psicométrica dos Instrumentos de pesquisa}

A análise da confiabilidade interna dos resultados, calculada através do coeficiente de Cronbach para as respostas obtidas com o WHOQOL-Bref, determinou um índice de 0.821, variando entre 0.801 (satisfação com a capacidade para realizar as tarefas cotidianas) e 0.838 (satisfação com os meios de transportes). Estes coeficientes foram considerados adequados aos valores adotados .

O cálculo do coeficiente de Cronbach para as respostas do IPAQ-Long considerou apenas os itens estandardizados, determinando deste modo um índice de 0.770. Justifica-se este procedimento pelas características psicométricas do instrumento, que permite respostas que podem variar de 1 a 7 (dias por semana) e 0 a 960 (tempo de realização da atividade em minutos) impondo variâncias elevadas. 
Descrição dos dados sócio-demográficos, ocupacionais, antropométricos e de saúde

Os dados sócio-demográficos e ocupacionais caracterizam os sujeitos em sua maioria como adultos, casados, com escolarização ao nível do ensino médio, com mais de 20 anos de atuação na Polícia Militar ( $\mathrm{X}=15.1$ anos), que não desenvolve nenhuma outra atividade ocupacional (Tabela 1). Os dados antropométricos e de saúde caracterizam os sujeitos em sua maioria na faixa recomendável de peso calculada pelo índice de massa corporal, que possui boa percepção de sua saúde e com alto nível de atividade física e baixo risco para doenças cardiovasculares. (Tabela 2). O valor médio da massa corporal dos sujeitos foi $80.2 \mathrm{~kg}$ e o da estatura corporal foi de $174 \mathrm{~cm}$.

Tabela 1

Variáveis sócio-demográficas e ocupacionais de Policiais Militares, Santa Catarina, $2011(n=302)$.

\begin{tabular}{|c|c|}
\hline Variável & $n(\%)$ \\
\hline \multicolumn{2}{|l|}{ Idade (anos) } \\
\hline $19-24$ & $69(22.8)$ \\
\hline $25-44$ & $173(57.3)$ \\
\hline$>44$ & $60(19.9)$ \\
\hline \multicolumn{2}{|l|}{ Escolaridade } \\
\hline Fundamental & $28(9.3)$ \\
\hline Médio & $178(58.9)$ \\
\hline Superior & $86(28.5)$ \\
\hline Pós-Graduação & $10(3.3)$ \\
\hline \multicolumn{2}{|l|}{ Estado Civil } \\
\hline Solteiro & $74(24.5)$ \\
\hline Casado & $175(57.9)$ \\
\hline União estável & $30(9.9)$ \\
\hline Separado & $10(3.3)$ \\
\hline Divorciado & $13(4.3)$ \\
\hline \multicolumn{2}{|l|}{ Tempo de Atuação (anos) } \\
\hline $1-5$ & $104(34.4)$ \\
\hline $6-10$ & $10(3.3)$ \\
\hline $11-15$ & $14(4.6)$ \\
\hline $16-20$ & $20(6.6)$ \\
\hline$>20$ & $154(51)$ \\
\hline \multicolumn{2}{|c|}{ Outras Atividades Ocupacionais } \\
\hline Não respondeu & $61(20.2)$ \\
\hline Não desenvolve & $166(55)$ \\
\hline Desenvolve & $75(24.8)$ \\
\hline
\end{tabular}

Tabela 2

Variáveis antropométricas e de saúde de Policiais Militares, Santa Catarina, $2011(n=302)$.

\begin{tabular}{|c|c|}
\hline Variável & $n(\%)$ \\
\hline \multicolumn{2}{|l|}{ Massa (kg) } \\
\hline$<65$ & $24(7.9)$ \\
\hline $66-75$ & $106(35.1)$ \\
\hline $76-85$ & $86(28.5)$ \\
\hline $86-95$ & $58(19.2)$ \\
\hline$>95$ & $28(9.3)$ \\
\hline \multicolumn{2}{|l|}{ Estatura $(\mathrm{cm})$} \\
\hline $160-170$ & $109(36.1)$ \\
\hline $171-180$ & $148(49)$ \\
\hline $181-190$ & $43(14.2)$ \\
\hline$>190$ & $2(0.7)$ \\
\hline \multicolumn{2}{|l|}{ Índice de Massa Corporal } \\
\hline Baixo peso & $43(14.2)$ \\
\hline Peso recomendado & $117(38.7)$ \\
\hline Sobrepeso & $88(29.1)$ \\
\hline Obesidade I & $50(16.6)$ \\
\hline Obesidade II & $2(0.7)$ \\
\hline Obesidade III & $2(0.7)$ \\
\hline \multicolumn{2}{|l|}{ Perceção do Estado de Saúde } \\
\hline Muito Ruim & $1(0.3)$ \\
\hline Ruim & $8(2.6)$ \\
\hline Nem Ruim/Nem Bom & $31(10.3)$ \\
\hline Bom & $173(57.3)$ \\
\hline Muito Bom & $89(29.5)$ \\
\hline \multicolumn{2}{|l|}{ Risco a Saúde } \\
\hline Alto Risco & $12(4)$ \\
\hline Risco significante & $17(5.6)$ \\
\hline Risco Reduzido & $23(7.6)$ \\
\hline Baixo risco & $250(82.8)$ \\
\hline
\end{tabular}

\section{Descrição dos valores medianos das ativida- des físicas em Equivalente Metabólico}

$\mathrm{Na}$ Tabela 3 apresentam-se os dados descritivos dos Equivalentes Metabólicos (MET'S) relacionados aos tipos e domínios de atividade física dos Policiais Militares. A partir dos resultados apresentados na Tabela 3, pode-se constatar que os valores medianos do domínio das atividades físicas ocupacionais, das atividades físicas do tipo moderadas e do total de atividades físicas estão acima do recomendado para saúde e o valor mediano do domínio das atividades físicas de transporte se encontra abaixo do mínimo do recomendado para saúde. Os demais domínios e tipos de atividades físicas 
estão dentro dos limites recomendados para a saúde.

Tabela 3

Dados descritivos das atividades físicas de Policiais Militares, Santa Catarina, $2011(n=302)$.

\begin{tabular}{ccccc}
\hline $\begin{array}{c}\text { Atividade Física } \\
\text { (MET's) }\end{array}$ & Md & DP & P25 & P75 \\
\hline Domínio ocupacional & 3390 & 4875 & 1197 & 7246 \\
Domínio do transporte & 297 & 1404 & 0 & 795 \\
Domínio doméstico & 1320 & 3750 & 360 & 3004 \\
Domínio do lazer & 1306 & 2269 & 75 & 2597 \\
Tipo caminhada & 1584 & 1663 & 792 & 2673 \\
Tipo moderada & 3182 & 4936 & 480 & 4560 \\
Tipo vigorosa & 1920 & 3603 & 4180 & 13006 \\
Total & 7704 & 8352 & 1374 & 5491 \\
\hline
\end{tabular}

\section{Descrição dos valores medianos dos escores de qualidade de vida}

Na Tabela 4 apresenta-se os dados descritivos associados aos escores dos domínios da qualidade de vida dos Policiais Militares. Com base nos resultados apresentados na Tabela 4, pode-se verificar que o maior escore refere-se ao domínio social e o menor escore refere-se ao domínio ambiental. A maioria dos sujeitos, de acordo com questão do WHOQOL-Bref que não é computada no escore geral de qualidade de vida, considera boa sua qualidade de vida ( $n$ $=197)$.

\section{Tabela 4}

Dados descritivos da qualidade de vida de Policiais Militares, Santa Catarina, $2011(n=302)$.

\begin{tabular}{ccccc}
\hline $\begin{array}{c}\text { Escore de Qualidade } \\
\text { de Vida }\end{array}$ & $\mathrm{Md}$ & $\mathrm{DP}$ & $\mathrm{P} 25$ & $\mathrm{P} 75$ \\
\hline Domínio Físico & 57.1 & 11.5 & 50 & 64.3 \\
Domínio Psicológico & 62.5 & 11.5 & 58.3 & 70.8 \\
Domínio Social & 75 & 18.1 & 58.3 & 83.3 \\
Domínio Ambiental & 53.1 & 11.1 & 46.9 & 59.4 \\
Geral & 61.4 & 10.2 & 56 & 67.8 \\
\hline
\end{tabular}

\section{Análise multivariada das variáveis relaciona- das à qualidade de vida}

A Tabela 5 descreve os parâmetros da regressão múltipla dos escores de qualidade de vida segundo variáveis de atividade física, demográficas, ocupacionais e antropométricas.
Após a finalização da regressão simples de cada uma das variáveis independentes em relação à variável dependente foram selecionadas, para a entrada no modelo múltiplo, as variáveis: atividades físicas moderadas; atividades físicas de lazer; atividades físicas de transporte; atividades físicas domésticas; total das atividades físicas; nível de atividade física; idade; situação marital; tempo de serviço; peso; estatura; e índice de massa corporal, todas com valor de $p$ menor que 0.20 .

Tabela 5

Parâmetros da regressão linear múltipla das variáveis que permaneceram no modelo múltiplo e respetivos valores de $\beta$ e p de cada variável, Santa Catarina, 2009.

\begin{tabular}{ccc}
\hline Variável & $\beta^{*}$ & $\mathrm{p}^{* *}$ \\
\hline $\begin{array}{c}\text { Atividades físicas de lazer (por } \\
\text { aumento de 100 MET) }\end{array}$ & 0.09 & 0.001 \\
$\begin{array}{c}\text { Situação marital }(0=\text { casado, } \\
\text { vivendo com alguém; } 1=\text { solteiro, } \\
\text { separado, divorciado) }\end{array}$ & 3.70 & 0.003 \\
$\begin{array}{c}\text { Estatura (por aumento de } 10 \mathrm{~cm}) \\
\text { sem }\end{array}$ & 176.40 & 0.050
\end{tabular}

*: valor do coeficiente angular; ${ }^{* *}$ : valor da probabilidade de aceitação de erro tipo I

As variáveis atividades físicas de lazer, estatura e situação marital foram as que permaneceram no modelo múltiplo de regressão linear, todas com significância estatística $(p<.05)$. Dessa forma, Policiais Militares com maior atividade de lazer, maior estatura e que não vivem com alguém estão associados com maiores índices de qualidade de vida, independente de cada uma das variáveis independentes finais.

\section{DISCUSSÃO}

Deve-se informar que as limitações deste estudo estão associadas às pesquisas envolvidas com populações vulneráveis, neste caso: (a) a necessidade de considerar a não identificação nas respostas a determinadas variáveis; (b) perda amostral relacionada a participação integralmente voluntária, minorando assim, as influências da cadeia de comando presentes nas organizações militares. 
A partir do objetivo deste estudo foi possível analisar os dados de 302 Policiais Militares que atenderam aos critérios de elegibilidade e que foram coletados através do WHOQOL-Bref e do IPAQ-Long, os quais apresentaram consistência interna, validez e fidedignidade estatisticamente adequadas, demonstrando associações da qualidade de vida com a atividade física de lazer, a estatura e a situação marital, que são reforçadas pelo caráter aleatório imposto ao processo de amostragem adotado, e coadunadas, em parte, por estudos realizados com diversas populações.

A associação significante entre lazer e qualidade de vida tem sido identificada por diversos estudos (Lynch, Cerin, Owen, \& Aitken, 2007; Vuillemin et al., 2005; Wendel-Vos, Schuit, Tijhuis, \& Kromhout, 2004). Vuillemin et al. (2005), ao realizarem estudo na França, verificaram que, apesar dos poucos dados sobre a relação entre saúde, qualidade de vida e o tempo de atividade física de lazer na população francesa, os indivíduos que realizam atividade física de lazer tiveram melhores escores de qualidade de vida que aqueles que não fizeram. Wendel-Vos, Schuit, Tijhuis, e Kromhout (2004), ao realizarem pesquisa na Holanda, encontraram associações transversais entre atividades físicas de lazer moderadas e o estado geral de saúde, a vitalidade, o funcionamento físico e limitações do papel devido a problemas de saúde física. Entretanto, Wendel-Vos, Schuit, Tijhuis, e Kromhout (2004) identificaram associações longitudinais positivas somente para os componentes de saúde mental relacionados à qualidade de vida. A associação entre lazer e qualidade de vida não foi identificada somente em indivíduos saudáveis, mas também em portadores de diversas patologias, como por exemplo, no estudo de Lynch, Cerin, Owen, e Aitken (2007), desenvolvido na Austrália, que constataram que mesmo as pequenas alterações nas atividades físicas de lazer estão associadas à melhoria da qualidade de vida.

Os estudos sobre crescimento e desenvol- vimento da população em geral têm demonstrado que a estatura menos elevada está associada ao fracasso escolar (Wake, Coghlan, \& Hesketh, 2000), à diminuição das relações pessoais (Hensley \& Cooper, 1987) e às baixas condições de saúde (Asao et al., 2006), entre outros, enquanto a estatura mais elevada está associada, por exemplo, ao sucesso profissional (Hensley \& Cooper, 1987). Entretanto, apesar das variáveis apontadas estarem relacionadas à qualidade de vida, pesquisadores têm demonstrado que existem poucas evidências sobre o impacto integral da estatura na qualidade de vida dos indivíduos (Christensen, Djurhuus, Clayton, \& Christiansen, 2007).

Segundo estudos levantados, a situação marital pode estar associada com a qualidade de vida dos indivíduos (Ehsanpour, Eivazi, \& Davazdah-Emami, 2007; Ferreira \& Santana, 2003). Ehsanpour, Eivazi, e Davazdah-Emami (2007), em estudo realizado no Iran, constataram que os maiores escores da qualidade de vida estavam associados tanto aos indivíduos casados, quanto aos indivíduos divorciados divergindo, deste modo, dos resultados encontrados nesta pesquisa. Já Ferreira e Santana (2003), em estudo realizado em Portugal, verificaram que os indivíduos solteiros apresentam índices de qualidade de vida maiores que indivíduos casados, coadunando assim os resultados encontrados neste estudo.

\section{CONCLUSÕES}

Pode-se concluir que os Policiais Militares investigados apresentam os maiores escores no domínio social e os menores no domínio ambiental de qualidade de vida, possuem níveis de atividade física acima do recomendado, são casados, são escolarizados e estão dentro dos índices de peso recomendados. Pode-se concluir, ainda, que existem associações da qualidade de vida com as atividades físicas de lazer, a estatura e a situação marital. Entretanto, sugere-se que sejam ampliados e aprofundados estudos sobre qualidade de vida e 
atividade física em Policiais Militares.

\section{Agradecimentos:}

Deve-se expressar agradecimentos ao Governo do Estado de Santa Catarina, à Secretaria de Segurança Pública, à Corporação da Polícia Militar de Santa Catarina, aos Comandos dos batalhões e aos Policiais que participaram voluntariamente deste estudo.

\section{Conflito de Interesses:}

Nada declarado.

Financiamento:

Nada declarado.

\section{REFERÊNCIAS}

Asao, K., Kao, W. H., Baptiste-Roberts, K., Bandeen-Roche, K., Erlinger, T. P., \& Brancati, F. L. (2006). Short stature and the risk of adiposity, insulin resistance, and type 2 diabetes in middle age: The Third National Health and Nutrition Examination Survey (NHANES III) 1988-1994. Diabetes Care, 29, 1632-1637.

Barbetta, P. A. (2003). Estatística aplicada às ciências sociais. Florianópolis: EDUFSC.

Berg, A .M., Hem, E., Lau, B., \& Ekeberg, Ǿ. (2005). Help-seeking in the Norwegian Police Service. Journal of Occupational Health, 48, 145-153.

Resolução n. 196. (1996, 10 de outubro). Estabelece as diretrizes e normas regulamentadoras de pesquisas envolvendo seres humanos. Recuperado em 9 de outubro, 2009, de http:// conselho.saude.gov.br/docs/reso196.doc.

Christensen, T. L., Djurhuus, C. B., Clayton, P., \& Christiansen, J. S. (2007). An evaluation of the relationship between adult height and health-related quality of life in the general UK population. Clinical Endocrinology, 67(3), 407-412.

Craig, C. L., Marshall, A. L., Sjöström, M., Bauman, A. E., Booth, M. L., Ainsworth, B. E., Pekka, O. (2003). International Physical Activity Questionnaire: 12-country reliability and validity. Medicine and Science in Sports and Exercise, 35(8), 1381-1395.

Deschamps, F., Paganon-Badinier, I., Marchand, A. C., \& Merle, C. (2003). Sources and assessment of occupational stress in the police. Journal of Occupational Health, 45, 358-364.

Diniz, D., \& Corrêa, M. (2001). Declaração de Helsinki: relativismo e vulnerabilidade. Cadernos de Saúde Pública, 17(3), 679-688.

Ehsanpour, S., Eivazi, M., \& Davazdah-Emami, S. (2007). Quality of life after the menopause and its relation with marital status. Iranian Journal of Nursing and Midwifery Research, 12 (4), 130-135.

Farquhar, M. (1995). Definitions of quality of life: a taxonomy. Journal of Advanced Nursing, 22, 502-508.

Ferreira, P. L., \& Santana, P. (2003). Percepção de estado de saúde e de qualidade de vida da população activa: contributo para a definição de normas portuguesas. Revista Portuguesa de Saúde Pública, 21 (2), 15-30.

Fleck, M. P. A., Leal, O. F., Louzada, S., \& Xavier, M. (1999). Development of the Portuguese version of the WHO evaluation instrument of quality of life. Revista Brasileira de Psiquiatria, 21 (1), 19-28.

Fleck, M. P. A., Louzada, S., \& Xavier, M. (2000). Aplicação da versão em português do instrumento abreviado de avaliação da qualidade de vida "WHOQOL-bref". Revista de Saúde Pública, 34(2), 178-183.

Forattini, O. P. (1992). Ecologia, epidemiologia e sociedade. São Paulo: Artes Médicas.

Gill, T. M., \& Feinstein, A. R. (1994). A Critical Appraisal of the Quality of Quality-of-Life Measurements. The Journal of the American Medical Association, 272(8), 619-626.

Harper, A., \& Power, M. (1998). Development of the World Health Organization WHOQOL - BREF Quality of Life Assessment. Psychological Medicine, 28(3), 551-558.

Hensley, W. E., \& Cooper, R. (1987). Height and occupational success: a review and critique. Psychological Reports, 60, 843-849.

Institute for Social Research. (2005). Rapid Risk Factor Surveillance System. Recuperado em 15 de janeiro, 2009, de www.rrfss.on.ca/resources/ Physical\%20Activity.doc.

Karolinska Institutet. (2005). Guidelines for data processing and analysis of the International Physical 
Activity Questionnaire (IPAQ) - Short form: Scoring protocol. Recuperado em 25 de fevereiro, 2009, de http://www.ipaq.ki.se/ipaq.htm.

Leavell, H. R., \& Clark, E. G. (1978). Medicina preventiva. São Paulo: McGraw Hill do Brasil.

Loo, R. (2004). A typology of burnout types among police managers. Policing, 27(2), 156-165.

Lynch, B. M., Cerin, E., Owen, N., \& Aitken, J. F. (2007). Associations of leisure-time physical activity with quality of life in a large, population-based sample of colorectal cancer survivors. Cancer Causes Control, 18(7), 735-742.

Matsudo, S., Araujo, T., \& Matsudo, V. (2001). Questionário internacional de atividade física (IPAQ): estudo de validade e reprodutividade no Brasil. Revista Brasileira de Atividade Física \& Saúde, 6(2), 5-18.

Meeberg, G. A. (1993). Quality of life: a concept analysis. Journal of Advanced Nursing, 18(1), 32-38.

Pardini, R., Matsudo, S., \& Araújo, T. (2001). Validação do questionário internacional de nível de atividade física (IPAQ - versão 6): estudo piloto em adultos jovens brasileiros. Revista Brasileira de Ciência e Movimento, 9(3), 45-51.

Pereira, M. G. (2002). Epidemiologia: teoria e prática. Rio de Janeiro: Guanabara Koogan.

Polícia Militar de Santa Catarina. (2008). Recuperado em 17 de maio, 2009, de http://www.pm.sc.gov.br/website/redir. php? site $=40 \&$ act $=1 \& \mathrm{id}=4 \&$ url $=4$.

Sallis, J. F., \& Owen, N. (1999). Physical activity and behavioral medicine. Thousands Oaks: Sage Publications.

Sirgy, J. M. (2001). Handbook of quality-of-life research: an ethical marketing perspective. Boston: Kluwer.

Sorensen, L., Smolander, J., Louhevaara, V., Korhonen, O., \& Oja, P. (2000). Physical activity, fitness and body composition of Finnish police officers: a 15-year follow-up study. Occupational Medicine, 50(1), 3-10.
Thomas, J. R., \& Nelson, J. K. (2001). Research methods in physical activity. Champaign: Human Kinetics.

Smith, T. C., Zamorski, M., Smith, B., Riddle, J. R., LeardMann, C. A., Wells, T. S., Millennium Cohort Study Team (2007). The physical and mental health of a large military cohort: baseline functional health status of the Millennium Cohort. BMC Public Health, 7, 340.

Vuillemin, A., Boini, S., Bertrais, S., Tessier, S., Oppert, J. M., Hercberg, S., Briançon, S. (2005). Leisure time physical activity and health-related quality of life. Preventive Medicine, 41 (2), 562-569.

Wake, M., Coghlan, D., \& Hesketh, K. (2000). Does height influence progression through primary school grades? Archives of disease in childhood, 82, 297-301.

Wendel-Vos, G. C. W., Schuit, A. J., Tijhuis, M. A. R., \& Kromhout D. (2004). Leisure time physical activity and health-related quality of life: cross-sectional and longitudinal associations. Quality of Life Research, 13(3), 667-677.

World Health Organization. (1995). Physical status: the use and interpretation of anthropometry. Recuperado em 10 de dezembro, 2008, de http:// whqlibdoc.who.int/trs/WHO_TRS_854.pdf.

World Health Organization. (1996). WHOQOL-bref: introduction, administration, scoring and generic version of the assessment. Recuperado em $10 \mathrm{de}$ dezembro, 2008, de http://www.who.int/ mental_health/media/en/76.pdf.

World Medical Association. (1997). Declaration of Helsinki: recommendation guiding physicians in biomedical research involving human subjects. The Journal of the American Medical Association, 277, 925-926. 